\title{
CONTRIBUCIÓN DE POLIQUETOS (ANNELIDA: POLYCHAETA) EN LA DIETA DE TRES ESPECIES DE NOTOTÉNIDOS (PERCIFORMES: NOTOTHENIOIDEI) EN LA REGIÓN DE MAGALLANES.
}

\author{
CONTRIBUTION OF POLYCHAETES (ANNELIDA: POLYCHAETA) IN \\ THE DIET OF THREE NOTOTHENIOIDS SPECIES (PERCIFORMES: \\ NOTOTHENIOIDEI) FROM THE MAGELLAN REGION.
}

Mathias Hüne $e^{1} \&$ Gladys Rivera ${ }^{2}$

\begin{abstract}
The importance of polychaeta in the diet of three notothenioids of western Patagonia we determined. By summarizing information published, regarding Patagonotothen tessellata, P. cornucola and Harpagifer bispinis. An analysis of the percentage frequency of occurrence and numerical was performed, considering also the selectivity of prey by size. Nereid polychaeta were the only prey item present in all samples taken, showing high values in percentage frequency of occurrence in the Capitán Aracena Island (72.2\%) and 46.2\% in Puerto Williams referring $P$. tessellata and $H$. bispinis respectively. While for P. cornucola, Nereidae exhibited a frequency of occurrence in Puerto Riquelme of 5.9\% and 13.6\% in Puerto Williams. The analysis of prey selectivity by size indicated that Polynoidae was the largest prey item followed by Nereidae.
\end{abstract}

Key words: Patagonotothen tessellata, Patagonotothen cormucola, Harpagifer bispinis, stomacal contents.

1 Programa de Magíster en Ciencias mención Manejo y Conservación de Recursos Naturales en Ambientes Subantárticos, Facultad de Ciencias, Universidad de Magallanes, Casilla 113D, Punta Arenas, Chile. mathiashune@gmail.com

2 Instituto de Biología Marina, Universidad Austral de Chile, Casilla 567, Valdivia, Chile. 


\section{RESUMEN}

Se determinó la importancia de los poliquetos en la dieta de tres nototénidos de la Patagonia occidental. Utilizando información de una base de datos de contenido estomacal de Patagonotothen tessellata, P. cornucola y Harpagifer bispinis. Se realizó el análisis del porcentaje de la frecuencia de ocurrencia y numérica, considerando además la selectividad de presas por tamaño.

Los poliquetos Nereidos fueron el único ítem presa presente en todos los muestreos realizados, presentando altos valores porcentuales en su frecuencia de ocurrencia en la isla Capitán Aracena (72,2\%) y $46,2 \%$ en puerto Williams para $P$. tessellata y $H$. bispinis respectivamente. Mientras que para $P$. cornucola, Nereidae exhibió una frecuencia de ocurrencia en puerto Riquelme de 5,9\% y en puerto Williams de 13,6\%. El análisis de selectividad de presas por tamaño indicó que Polynoidae fue el ítem presa de mayor tamaño seguido por Nereidae.

Palabras clave: Patagonotothen tessellata, Patagonotothen cormucola, Harpagifer bispinis contenido estomacal.

\section{INTRODUCCIÓN}

Los peces nototénidos exhiben un rol clave como predadores, ya que ellos ocupan la mayoría de los nichos tróficos disponibles en el ecosistema, permitiéndoles alimentarse tanto en el bentos como en la columna de agua (Eastman \& DeVries 1982, Eastman 1993, Eastman \& McCune 2000). Dos de las especies más abundantes del sector intermareal en los canales patagónicos de la región de Magallanes son Patagonotothen cornucola (Richardson, 1844) y Harpagifer bispinis (Schneider, 1801) (Lloris \& Rucabado, 1991, Sielfeld \& Vargas, 1999); mientras que el submareal somero (menos de 15 metros de profundidad) se caracteriza por la abundancia de Patagonotothen tessellata (Richardson, 1845) (Sielfeld et al. 2006). Estas tres especies de nototénidos presentan hábitos bentónicos, asociados principalmente a los fondos de arena $(P$. tessellata) y rocoso $(P$. cornucola y $H$. bispinis) (Moreno \& Jara 1984, Laptikhovsky 2004).

Los poliquetos componen en general uno de los taxa más significativo en las comunidades marinas de fondos blandos y duros en cuanto a número de especies e individuos (Cañete et al. 1999, Ríos \& Mutschke 1999, Ríos et al. 2003). Sin embargo, la información disponible sobre la dieta de los peces nototénidos que reportan a los poliquetos como presa es escasa (Guzmán \& Campodónico 1973, Moreno \& Jara 1984, Murillo \& Oyarzún 2002, Laptikhovsky 2004, Laptikhovsky \& Arkhipkin 2003, Hidalgo et al. 2007, Martin \& Bastida 2008). Los estudios disminuyen aun más al presentar información a nivel taxonómico de orden, familia o especie (Murillo \& Oyarzún 2002, Hidalgo et al. 2007, Martin \& Bastida 2008). Murillo \& Oyarzún (2002) indican que Patagonotothen longipes (Steindachner 1876), en el Fiordo de Castro (isla de Chiloé), presentó el poliqueto Nereis sp. en su contenido estomacal, aunque en muy bajo número. Siendo también reportado por Hidalgo et al. (2007) en la dieta de $P$. cornucola en el cabo dos Bahías (Provincia de Chubut, Argentina) junto a Eunice sp., formando parte en la alimentación de este pez. Mientras que los poliquetos de la familia Maldanidae, constituyen parte de la dieta de Eleginops maclovinus (Cuvier 1830) en la ría Deseado (Provincia de Santa Cruz; Argentina) (Martin \& Bastida 2008).

En este trabajo se reporta la importancia del grupo poliquetos en la dieta de tres especies de nototénidos, considerando el porcentaje de la frecuencia de ocurrencia, numérica y las interacciones tróficas basadas en el tamaño de predadores y presas, mediante el índice de selectividad de presas por tamaño.

\section{MATERIAL Y MÉTODOS}

Se utilizó información de una base de datos de contenido estomacal de tres especies de nototénidos. La base de datos fue establecida a partir de muestreos en tres áreas: estero Staples (5402'S, $71^{\circ} 16^{\prime} \mathrm{W}$ ) en la isla Capitán Aracena; Puerto Riquelme (51 $\left.49^{\circ} \mathrm{S}, 72^{\circ} 38^{\prime} \mathrm{W}\right)$ en el golfo Almirante Montt

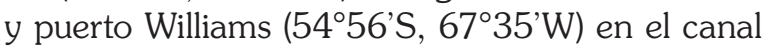
Beagle (Fig. 1). Las muestras fueron tomadas entre 


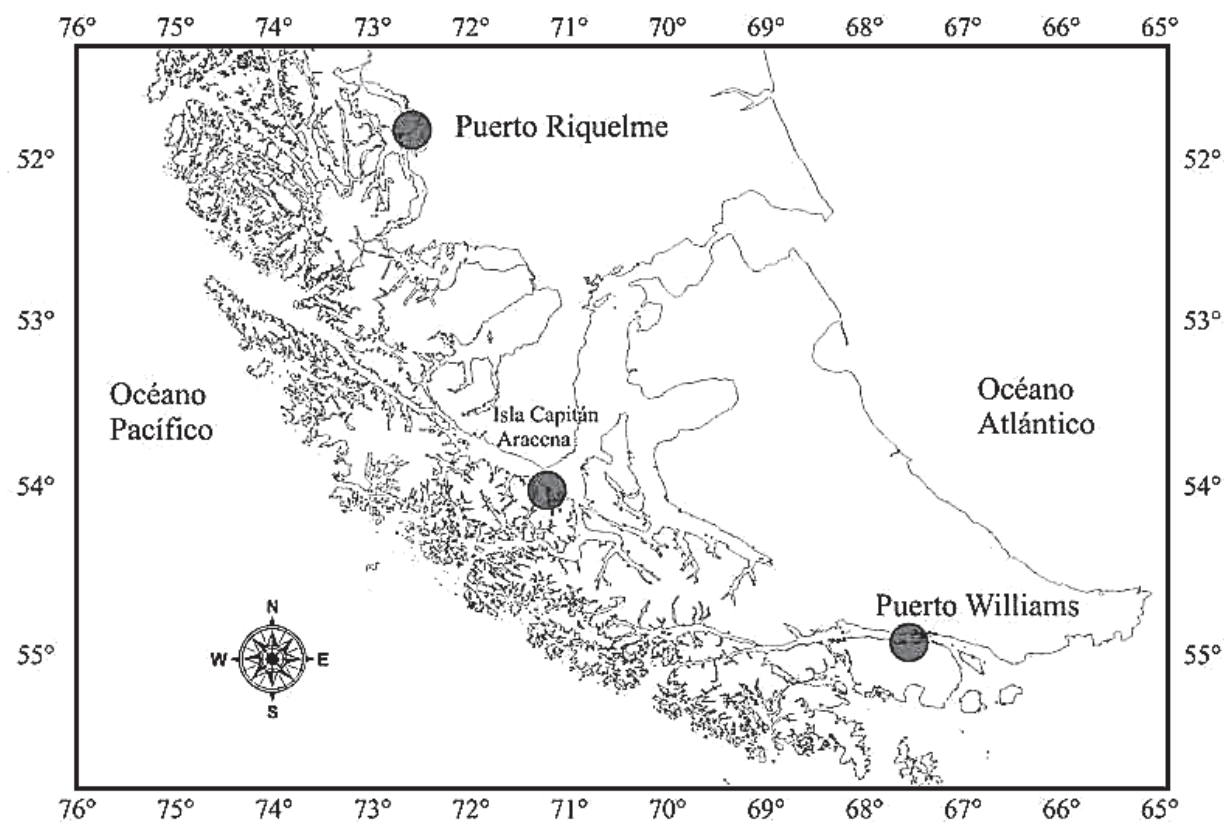

Fig. 1. Áreas de muestreo de los ejemplares de peces.

el 10 de marzo de 2007 y el 23 de septiembre de 2008 (Tabla 1).

Los peces fueron capturados mediante una red agallera (60 $\mathrm{m}$ de largo, $3 \mathrm{~m}$ de alto y $25 \mathrm{~mm}$ de tamaño de malla) para $P$. tessellata y se realizó una colecta manual para $P$. cornucola y $H$. bispinis. A cada ejemplar capturado se le midió la longitud total $(\mathrm{mm})$ mediante un pie de metro digital de 0,01 $\mathrm{mm}$ de precisión y el peso total (g) con una balanza digital de $0,1 \mathrm{~g}$ de exactitud. Posteriormente se realizó la disección del ejemplar, extrayéndose el estómago, el cual fue preservado en una solución de etanol al $70 \%$ para su posterior análisis. En laboratorio, los estómagos fueron analizados individualmente bajo una lupa estereoscópica Zeiss Stemi SR y los contenidos fueron separados para el recuento y pesaje de las presas. La identificación de los poliquetos presentes en los contenidos se realizó utilizando literatura especializada como Rozbaczylo (1980), Montiel et al. (2004), Rozbaczylo et al. (2006a), Rozbaczylo et al. (2006b). El peso húmedo de todos los ejemplares de poliquetos encontrados en los contenidos estomacales fueron obtenidos mediante una balanza analítica marca Denver Instrument, modelo AA-200DS, con 0,0001 g de precisión y la longitud total se obtuvo mediante un ocular graduado con precisión de $0,01 \mathrm{~mm}$. El análisis del contenido estomacal se realizó mediante los métodos de frecuencia (número de ejemplares que consumen un cierto ítem alimentario) y numéricos (cantidad de organismos consumidos de cada ítem alimentario), expresados en porcentaje (Hyslop 1980).

TABLA 1. Fecha, lugar de captura, número de estómagos analizados y número de estómagos con contenido estomacal, entre el 10 de marzo de 2007 y el 23 de septiembre de 2008.

\begin{tabular}{cccccc}
\hline Muestreo & Fecha & Lugar & Especie & Estómagos analizados & $\begin{array}{c}\text { Estómagos con } \\
\text { contenido }\end{array}$ \\
\hline 1 & $10-11 / 03 / 07$ & Isla Capitán Aracena & P. tessellata & 126 & 97 \\
2 & $10-11 / 04 / 07$ & Puerto Riquelme & P. tessellata & 112 & 98 \\
3 & $8-9 / 05 / 07$ & Puerto Riquelme & P. cornucola & 80 & 51 \\
4 & $22-23 / 09 / 08$ & Puerto Williams & P. cornucola & 24 & 22 \\
5 & $15-16 / 08 / 07$ & Puerto Riquelme & H. bispinis & 42 & 39 \\
6 & $22-23 / 09 / 08$ & Puerto Williams & H. bispinis & 75 & 65 \\
\hline
\end{tabular}


La selectividad de presas por tamaño se determinó mediante la metodología de Ursin (1973) para establecer la razón óptima entre el peso del predador y el peso de la presa, para lo cual se calcula el tamaño de cada presa en el estómago de cada predador como la razón entre el peso del predador y el peso de la presa, designando a cada razón como un valor ISU. Exhibiendo las preferencias como una distribución de frecuencias derivada del logaritmo natural de sus pesos cuya formula es:

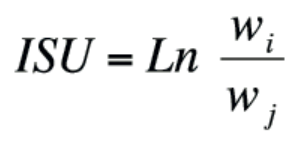

donde $\mathrm{w}_{\mathrm{i}}$ es el peso del predador $\mathrm{i}(\mathrm{g}) ; \mathrm{w}_{\mathrm{i}}$ es el peso del taxon de presa j (g) en el estómago del predador i. Los valores ISU se agruparon en intervalos de 1,0 y graficados por especies-presa para los sitios de muestreo. El promedio del ISU refleja el tamaño promedio entre el predador y sus presas y la desviación estándar es una medida relativa de la selectividad, siendo inversamente proporcional a ésta.

\section{RESULTADOS}

Se analizó un total de 459 estómagos, donde el 81\% (372 estómagos) tenían contenido. De éstos, 238 estómagos pertenecían a $P$. tessellata que presentó un $82 \%$ con contenido (Tabla 1). En la dieta de este pez se determinaron 8 familias de poliquetos para la isla Capitán Aracena, correspondientes a Nereidae, Spionidae, Polynoidae, Terebellidae, Arenicolidae, Cirratilidae, Orbiniidae y Nephtyidae (Tabla 2), siendo los poliquetos $\mathrm{Ne}$ reidos el ítem presa mas importante en términos del porcentaje de la frecuencia de ocurrencia (72\%) y numérica (55.2\%). Como se aprecia en la tabla 2 , el número de especie de poliquetos en puerto Riquelme disminuye a 2 familias pertenecientes a Nereidae y Lumbrineridae, presentando los Nereidos una frecuencia de ocurrencia y numérica de 15,3\% y $3,3 \%$ respectivamente.

De un total de 104 estómagos de P. cornucola (Tabla 1), 70,2\% presentaron contenido. Los cuales estuvieron conformados para Puerto Riquelme por 2 familias pertenecientes a Nereidae y Polynoidae,

TABLA 2, Poliquetos en la dieta de P. tessellata obtenidos en la isla Capitán Aracena ( $\mathrm{n}=97)$ y puerto Riquelme ( $\mathrm{n}=98)$, entre el 10 de marzo y 11 de abril de 2007. Porcentaje de la frecuencia de ocurrencia $(\% \mathrm{~F})$, porcentaje numérico $(\% \mathrm{~N})$ y longitud total (LT \pm desviación estandar) y peso ( $\mathrm{g} \pm$ desviación estándar).

\begin{tabular}{|c|c|c|c|c|c|c|c|c|}
\hline \multirow[b]{2}{*}{ Presas } & \multicolumn{4}{|c|}{ Isla Capitán Aracena } & \multicolumn{4}{|c|}{ Puerto Riquelme } \\
\hline & $\% \mathrm{~F}$ & $\% \mathrm{~N}$ & $\mathrm{LT}(\mathrm{mm})$ & Peso (g) & $\% \mathrm{~F}$ & $\% \mathrm{~N}$ & LT (mm) & Peso (g) \\
\hline Nereidae & 72,2 & 55,2 & $25,61 \pm 13,49$ & $0,08 \pm 0,10$ & 15,3 & 3,3 & $9,93 \pm 9,84$ & $0,01 \pm 0,02$ \\
\hline Spionidae & 4,1 & 0,9 & $26,75 \pm 6,18$ & $0,02 \pm 0,002$ & & & & \\
\hline Polynoidae & 6,2 & 1,7 & $38,14 \pm 17,57$ & $0,88 \pm 0,78$ & & & & \\
\hline Terebellidae & 1,0 & 0,2 & 31 & 0,38 & & & & \\
\hline Arenicolidae & 1,0 & 0,2 & 12 & 0,07 & & & & \\
\hline Cirratilidae & 1,0 & 0,2 & 10 & 0,002 & & & & \\
\hline Orbiniidae & 1,0 & 0,2 & 25 & 0,008 & & & & \\
\hline Nephtyidae & 1,0 & 0,2 & & & & & & \\
\hline Lumbrineridae & & & & & 7,1 & 1,7 & $11,23 \pm 2,95$ & $0,004 \pm 0,001$ \\
\hline Polychaeta sin identificar & 3,1 & 0,9 & $13,22 \pm 12,23$ & $0,06 \pm 0,007$ & & & & \\
\hline
\end{tabular}

TABLA 3. Poliquetos en la dieta de P. cornucola obtenidos en puerto Riquelme $(\mathrm{n}=51)$ y puerto Williams $(\mathrm{n}=$ 22) entre el 8 de mayo de 2007 y el 23 de septiembre de 2008. Porcentaje de la frecuencia de ocurrencia (\%F), porcentaje numérico $(\% \mathrm{~N})$ y longitud total (LT \pm desviación estandar) y peso ( $\mathrm{g} \pm$ desviación estándar).

\begin{tabular}{|c|c|c|c|c|c|c|c|c|}
\hline & \multicolumn{4}{|c|}{ Puerto Riquelme } & \multicolumn{4}{|c|}{ Puerto Williams } \\
\hline Presas & $\% \mathrm{~F}$ & $\% \mathrm{~N}$ & $\mathrm{LT}(\mathrm{mm})$ & Peso (g) & $\% \mathrm{~F}$ & $\% \mathrm{~N}$ & $\mathrm{LT}(\mathrm{mm})$ & Peso (g) \\
\hline Nereidae & 5,9 & 0,5 & $2,67 \pm 0,58$ & $0,00001 \pm 0$ & 13,6 & 2,5 & $4,33 \pm 1,53$ & $0,002 \pm 0,0016$ \\
\hline Polynoidae & 5,9 & 1,0 & 30 & 0,002 & & & & \\
\hline Polychaeta sin identificar & 2,0 & 0,1 & & & & & & \\
\hline
\end{tabular}


TABLA 4. Poliquetos en la dieta de H. bispinis obtenidos en puerto Riquelme $(\mathrm{n}=39)$ y puerto Williams ( $n=65$ ), entre el 15 de agosto de 2007 y el 23 de septiembre de 2008 . Porcentaje de la frecuencia de ocurrencia $(\% \mathrm{~F})$, porcentaje numérico $(\% \mathrm{~N})$ y principales atributos de las presas.

\begin{tabular}{lcccccccc}
\hline & \multicolumn{3}{c}{ Puerto Riquelme } & \multicolumn{3}{c}{ Puerto Williams } \\
\hline Presas & $\% \mathrm{~F}$ & $\% \mathrm{~N}$ & $\mathrm{LT}(\mathrm{mm})$ & Peso $(\mathrm{g})$ & $\% \mathrm{~F}$ & $\% \mathrm{~N}$ & LT $(\mathrm{mm})$ & Peso $(\mathrm{g})$ \\
\hline Nereidae & 20,5 & 2,0 & $5,36 \pm 5,61$ & $0,005 \pm 0,01$ & 46,2 & 8,4 & $14,27 \pm 14,62$ & $0,03 \pm 0,06$ \\
Polynoidae & 5,1 & 0,3 & $11,5 \pm 13,43$ & $0,022 \pm 0,03$ & 1,5 & 0,3 & 3 & 0,00002 \\
\hline
\end{tabular}

exhibiendo en ambas familias un 5,9\% de ocurrencia (Tabla 3), sin embargo en Puerto Williams solo se detectó la familia Nereidae, presentando el 13,6\% de ocurrencia en la dieta de P. cornucola.

Por otra parte, se analizaron 117 estómagos de H. bispinis (Tabla 1), de los cuales el 89\% exhibían contenido, presentando las familias Nereidae y Polynoidae y exhibiendo la primera los mayores valores porcentuales para su frecuencia de ocurrencia en la dieta de $H$. bispinis en ambos sitios de muestreo, con valores de $20,5 \%$ en puerto Riquelme y 46,2\% en puerto Williams (Tabla 4).

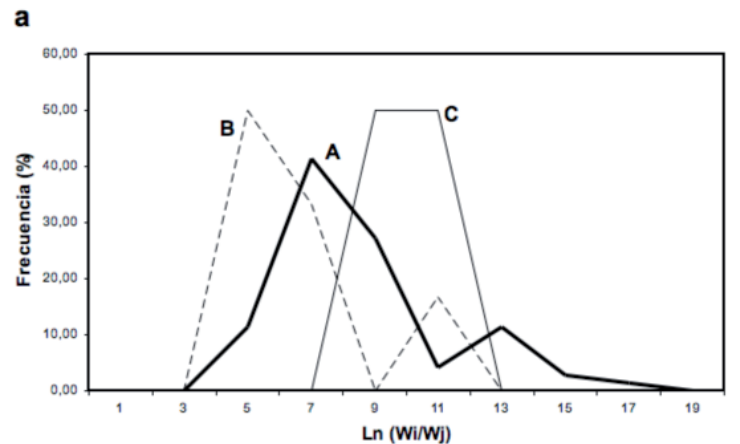

De acuerdo a los gráficos de distribuciones de frecuencias de los valores del índice de selectividad de Ursin (ISU) la familia Polynoidae resultó ser la presa de mayor tamaño en relación al peso del predador $(P$. tessellata) en la isla Capitán Aracena (Fig. 2), junto a Nereidae en la dieta de H. bispinis en puerto Williams (Fig. 3). Los valores del promedio y desviación estándar del ISU se presentan en la tabla 5. Donde se aprecian los menores valores promedio para Polynoidae y Nereidae para $P$. tessellata y $H$. bispinis respectivamente. Mientras que en puerto Williams, Nereidae tuvo una mayor

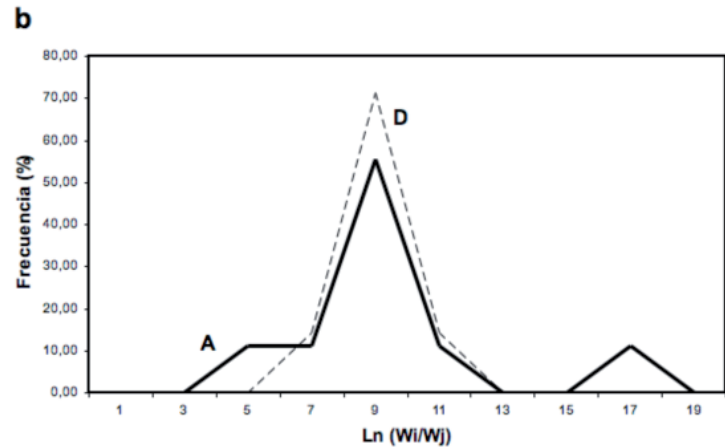

Fig. 2. Distribución de frecuencias del índice de selectividad de Ursin de los poliquetos en la dieta de P. tessellata en: a) Isla Capitan Aracena, y b) Puerto Riquelme. A: Nereidae, B: Polynoidae, C: Spionidae, y D: Lumbrineridae.

a

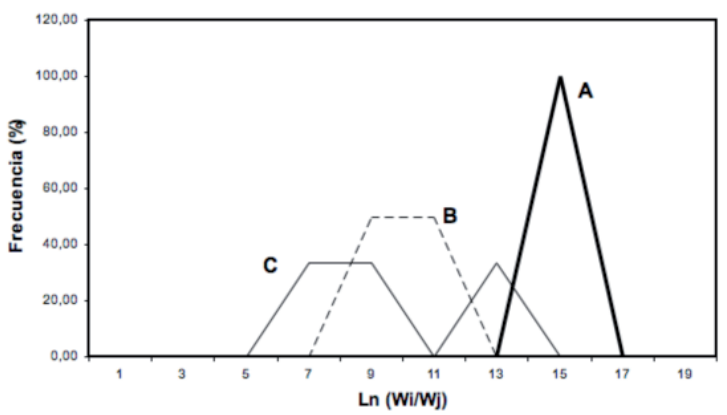

b

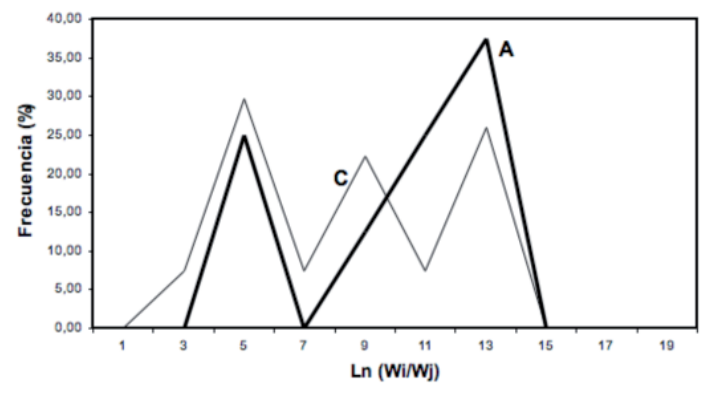

Fig. 3. Distribución de frecuencias del índice de selectividad de Ursin de los poliquetos en la dieta de $P$. cornucola y H. bispinis en puerto Riquelme y puerto Williams. a) $P$. cornucola, y b) $H$. bispinis. A: Nereidae y B: Polynoidae en puerto Riquelme, C: Nereidae en puerto Williams. 
TABLA 5. ISU promedios y desviaciones estándar (DE) de los ISU de los poliquetos en la dieta de $P$. tessellata en la isla Capitán Aracena y puerto Riquelme; P. cornucola en puerto Williams y puerto

Riquelme; $H$. bispinis en puerto Williams y puerto Riquelme. N: número de observaciones.

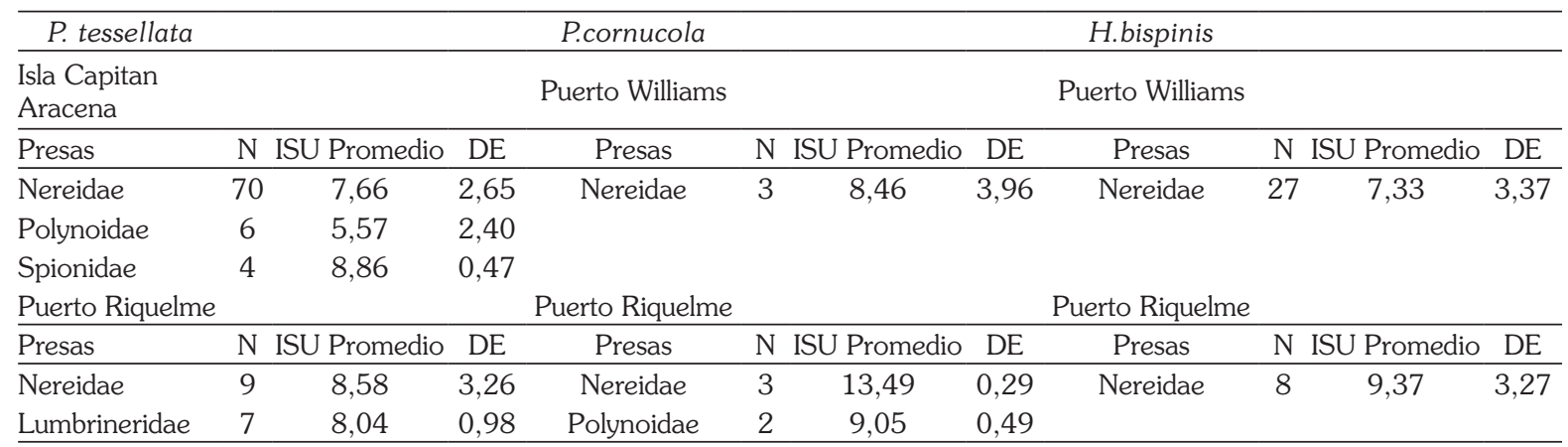

desviación estándar ( $\mathrm{DE}=3.96)$ (Tabla 5), lo que se traduce como una baja selección por tamaño por parte de P. cornucola por esta presa.

\section{DISCUSIÓN}

Los resultados indican que la composición de poliquetos presentes en la dieta de P. tessellata, $P$. cornucola y $H$. bispinis estuvo dominada preferentemente por Nereidae, el cual estuvo presente en todos los sitios de muestreo y fue el ítem presa principal para $P$. tessellata en la isla Capitán Aracena (Tabla 2) y H. bispinis en puerto Williams (Tabla 4). Es posible que la alta incidencia de Nereidae en la alimentación de $P$. tessellata en la isla Capitán Aracena, este relacionado a la fase pelágica reproductiva de estos poliquetos, ya que al analizar 19 individuos de Nereidae a nivel de especie, estos correspondieron a Platynereis australis (Schmarda 1861) en fase de epitoco (Montiel, comunicación personal). Considerando lo anterior, esto llevaría a suponer que $P$. tessellata dejaría su habitual nicho trófico para alimentarse de organismos pelágicos cuando están disponibles.

$P$. cornucola exhibió un menor consumo de poliquetos en comparación a los otros nototénidos analizados, en relación al porcentaje de la frecuencia de ocurrencia y numérica (Tabla 3). Esta situación se contrapone a la reportada por Hidalgo et al. (2007), donde este pez en el cabo dos Bahías (Provincia de Chubut, Argentina) presentó un 72,13\% de frecuencia de ocurrencia y 16,92\% de numérica para el ítem presa de los poliquetos. Sin embargo, el mayor porcentaje de estos métodos corresponde al ítem poliquetos sin identificar. Esta situación también fue observada por Moreno \& Jara (1984), los que registraron una alta frecuencia de ocurrencia en el consumo de poliquetos por parte de $P$. cornucola sin entregar mayor información taxonómica de este ítem presa.

Los resultados obtenidos para la selectividad de presas por tamaños de Ursin (1973) sugieren que P. tessellata en la isla Capitán Aracena, seleccionaría por tamaño a Polynoidae, ya que estos presentaron en promedio las mayores tallas $(38,14 \pm 17,57)$ (Tabla 2). Sin embargo, la más baja desviación estándar se presentó en Spionidae, lo que se interpretaría como una preferencia por este poliqueto por parte del predador. Por otro lado, los Nereidos en general no mostraron una distribución normal en el índice de selectividad en todos los sitios de muestreo, esto podría explicarse con el fundamento de Ursin (1973), el cual señala que la distribución de frecuencias del índice se aleja de una distribución normal cuando la abundancia de presas es alta en el medio. Desafortunadamente, la ausencia de datos distribuidos temporalmente a causa de la lejanía y difícil acceso a las zonas muestreadas, no posibilita una comparación de la variación estacional en la alimentación. No obstante, es positivo subrayar que el análisis de la dieta de estos peces no sólo permite establecer su trofodinámica, sino que también permite conocer los organismos que habitan en un lugar determinado donde éstos son consumidos, ya que muchas de estas presas no son extraídas por las artes de pesca, constituyendo por este motivo una apreciada fuente de información de uso tanto científico como biológico-pesquero. 


\section{AGRADECIMIENTOS}

Agradecemos a José Sánchez y Pedro Contreras por la ayuda prestada en terrenos y el apoyo logístico. A dos revisores anónimos por sus comentarios y contribuciones a este escrito.

\section{LITERATURA CITADA}

Cañete, J., G. Leighton \& F. Aguilera 1999. Polychaetes from Aysén Fjord, Chile: distribution, abundance and biogeographical comparison with the shallow sof-bottom polychaete fauna from Antarctica and the Magellan Province. Scientia Marina 63: 243-252.

Eastman, J. \& A. De Vries 1982. Buoyancy studies of notothenioid fishes in McMurdo Sound, Antarctica. Copeia 2: 385-393.

Eastman, J. 1993. Antarctic fish biology: evolution in a unique environment. Academia Press, San Diego, $322 \mathrm{pp}$.

Eastman, J. \& A. McCune 2000. Fishes on the Antarctic continental shelf: evolution of a marine species flock? Journal of Fish Biology 57 (Suppl A): 84-102.

Guzmán, L. \& I. Campodonico 1973. Algunos aspectos de la biología de Eleginops maclovinus (Cuv. y Val.1830), con especial referencia a su morfometría, caracteres merísticos y alimentación. Anales Instituto Patagonia (Chile) 4 (1-3): 344-371.

Hidalgo, F., B. Silliman, M. Bazterrica \& M. Bertness 2007. Predation on the Rocky Shores of Patagonia, Argentina. Estuaries and Coasts 30 (5): 886-894.

Hyslop, E.J. 1980. Stomach contents analysis - a review of methods and their application. Journal of Fish Biology 17: 411-429.

Laptikhovsky V. 2004. A comparative study of diet in three sympatric populations of Patagonotothen species (Pisces: Nototheniidae). Polar Biology, 27: 202-205.

Laptikhovsky, V. \& A. Arkhipkin 2003. An impact of seasonal squid migrations and fishing on the feeding spectra of subantarctic notothenioids Patagonotothen ramsayi and Cottoperca gobio around the Falkland Islands. Journal applied Ichthyology 19: 35-39.
Lloris, D. \& J. Rucabado 1991. Ictiofauna del Canal Beagle (Tierra del Fuego), aspectos ecológicos y análisis biogeográfico. Instituto Español de Oceanografía, Madrid, $182 \mathrm{pp}$.

Martin, J. \& R. Bastida 2008. Contribución de las comunidades bentónicas en la dieta del róbalo (Eleginops maclovinus) en la ría Deseado (Santa Cruz, Argentina). Revista de Biología Marina y Oceanografía 36 (1): 1-13.

Montiel, A., C. Ríos, E. Mutschke \& N. Rozbaczylo 2004. Poliquetos de fiordos y canales adyacentes al Campo de Hielo Patagónico Sur, Chile (Annelida: Polychaeta). Ciencia y Tecnología de Mar 27 (1): 49-67.

Moreno, C. \& F. Jara 1984. Ecological studies on fish fauna associated with Macrocystis pyrifera belts in the south of Fueguian Islands, Chile. Marine Ecology Progress Series 15: 99-107.

Murillo, C. \& C. Oyarzún 2002. Variación ontogenetica en la dieta de Patagonotothen longipes (Steindachner 1876) (Perciformes: Nototheniidae) en el fiordo de Castro, Chiloe. Gayana 66 (2): 219-224.

Ríos, C. \& E. Mutschke 1999. Community structure of intertidal boulder-cobble fields in the Strait of Magellan, Chile. Scientia Marina 63(Suppl. 1): 193-201.

Ríos, C., E. Mutschke \& E. Morrison 2003. Biodiversidad bentónica en el estrecho de Magallanes, Chile. Revista de Biología Marina y Oceanografía 38 (1): 1-12.

Rozbaczylo, N. 1980. Clave para el reconocimiento de Familias de Anélidos Poliquetos de Mar Chileno. Studies on Neotropical Fauna and Environment 15: 167-196.

Rozbaczylo, N., R. Moreno \& O. Díaz 2006a Poliquetos bentónicos submareales de fondos blandos de la Región de Aysen, Chile: Clados Terebellida (Annelida, Polychaeta). Ciencia y Tecnología del Mar 29(1): 70-90.

Rozbaczylo, N., R. Moreno \& O. Díaz 2006b Poliquetos bentónicos submareales de fondos blandos de la región de Aysén, Chile: Clados Amphinomida, Eunicida, Spionida, Sabellida y Scolecida (Annelida, Polychaeta). Investigaciones Marinas 34(1): 43-62.

Sielfeld, W., G. Guzman \& N. Amado 2006. Distribución de peces del litoral rocoso de los canales 
patagónicos occidentales (48³7'S - 5334'S). Ursin, E. 1973. On the prey size preference of cod Anales Instituto Patagonia (Chile) 34: 21-32. and dab. Meddelelser fra Danmarks FiskeriSielfeld, W. \& M. Vargas 1999. Review of Marine Zoogeography of Chilean Patagonia (42 og Havundersoegelser, 7: 85-98.

$57^{\circ}$ S). Scientia Marina 63 (Supl.1): 451-463. 